\title{
REVISIONES
}

\section{Aspectos críticos en las propuestas sobre una nueva arquitectura de la educación pública chilena*}

\author{
Critical issues proposals on a new architecture chilean education
}

\section{Questões críticas propostas sobre uma nova arquitetura educação chilena}

\author{
Donoso-Díaz, Sebastián ${ }^{a}$; Castro-Paredes, Moyra ${ }^{b}$; Alarcón-Leiva, Jorge ${ }^{c}$ \\ anstituto de Investigación y Desarrollo Educacional (IIDE), Universidad de Talca, Talca, Chile. \\ Teléfono: 56-71-2200253. Correo electrónico: sdonoso@utalca.cl \\ bInstituto de Investigación y Desarrollo Educacional (IIDE), Universidad de Talca, Talca, Chile. \\ Teléfono: 56-71-2200253. Correo electrónico: mocastro@utalca.cl \\ 'Instituto de Investigación y Desarrollo Educacional (IIDE), Universidad de Talca, Talca, Chile. \\ Teléfono: 56-71-2200253. Correo electrónico: joalarcon@utalca.cl
}

\begin{abstract}
RESUMEN
El texto tiene por finalidad analizar la relevancia que adquiere para la educación pública instalar una nueva institucionalidad, lo que se ha denominado una nueva arquitectura para el sector, que dé cuenta de las principales dificultades y limitaciones que ha presentado en más de tres décadas de funcionamiento, tras la reforma del año 1981 que sentó las bases de la institucionalidad vigente y de los problemas que acusa, marginalmente resueltos por la Ley General de Educación del año 2009. Entendiendo que esta cuestión reviste un carácter estratégico, después de identificar los puntos claves del diagnóstico, se revisan las propuestas desplegadas en el Informe del Consejo Asesor para la Calidad, y los debates que se han generado a partir de esta y las modalidades propuestas, finalizándose con la revisión de algunos de los tópicos críticos fundamentales que viabilizan los impactos de la instalación de la nueva institucionalidad.
\end{abstract}

Palabras clave: nueva institucionalidad, educación publica, elementos críticos, instalación de reformas.

\begin{abstract}
The text aims to analyze the implication, for public education, of installing a new institutional framework, called a new architecture for the sector, which notices the major difficulties and limitations in more than three decades of operation after the reform of 1981, setting the foundations for existing institutions and the problems that have followed, marginally been resolved by the Education Act 2009. Understanding that this issue is strategic in nature, after identifying the key points of diagnosis, proposals deployed in the Report of the Advisory Councils for Quality are reviewed, and the debates that have arisen from this and the proposed modalities. Finally, a review of some of the key critical issues that make possible the impacts of the installation of the new institutions are presented.
\end{abstract}

Key words: new institutionalism, public education, critical elements, installing reforms.

\section{RESUMO}

Objetiva-se analisar a importância adquirida para a educação pela instalação de uma nova institucionalidade, o que tem sido chamado de nova arquitetura do setor, que dê conta das principais dificuldades e limitações surgidas em mais de três décadas de operacionali-zação após a reforma de 1981, a qual estabeleceu as bases da institucionalidade vigente e dos problemas acusados em razão da Lei Geral da Educação de 2009. Entendendo que esta

* Estudio correspondiente a los proyectos Fondecyt 1120041, Conicyt Redes 120015 y Pia- Conicyt-Cie 05. 
questão é de natureza estratégica, depois de identificar os pontos-chave do diagnóstico, revisam-se as propostas implantadas pelo Informe dos Conselhos Assessores da Qualidade e os debates que surgiram a partir de este e das modalidades propostas. Finaliza com a revisão de alguns dos tópicos críticos fundamentais que viabilizam os impactos da instalação da nova institucionalidade.

Palavras chave: nova institucionalidade, educação pública, elementos críticos, instalação de reformas.

\section{PRESENTACIÓN}

El año 2011 se conformó un gran movimiento estudiantil que, finalmente, terminó siendo un poderoso movimiento social, el cual retomó las consignas de fortalecimiento de la educación pública de su predecesor, el movimiento de estudiantes secundarios del año 2006 (conocido como el movimiento pingüino), ampliando y profundizando las demandas y propuestas iniciales tras las consignas de educación pública gratuita y de calidad y de fin al lucro y a la municipalización en educación, resumiendo con ello buena parte de las complejas aspiraciones larvadas tras años de postergación de muchas inquietudes que en este plano se habían puesto en evidencia en la sociedad chilena desde mediados de la década pasada.

En los más de ocho años que han transcurrido desde el estallido del movimiento pingüino, se han sucedido un conjunto de acontecimientos y fenómenos relevantes, que si bien no han transformado radicalmente el escenario en sus aspectos centrales, son indispensables de considerar para comprender la importancia, profundidad y complejidad de la temática en análisis: las propuestas de nueva arquitectura de la educación pública nacional, como consecuencia de las demandas generadas en pro del fortalecimiento de la educación pública.

En los primeros años de democracia (década del 90) colisiona lo que Corvalán llama la "narrativa centro estatista", previa a 1973 con la "narrativa de mercado". Esta última es la que introduce las importantes transformaciones en los 80 y la que se sustenta en la "economía neoliberal como la principal e incluso única disciplina [científica] capaz de generar lenguaje explicativo y analítico del sistema educativo" (Corvalán, 2013: 68). De ese modo, la conformación de la agenda de política educativa pertenece al proceso de síntesis y construcción intelectual: un tiempo de consenso entre actores y policy makers que llevaría a las políticas de la coalición gobernante (la Concertación ${ }^{1}$ ) a ser una "prolongación maquillada de aquellas de inspiración neoliberal del gobierno militar" (Picazo, 2010: 64).

De esta manera, las políticas concertacionistas no lograron modificar sustancialmente el diseño institucional del sistema educacional chileno instaurado por la dictadura, a saber: descentralización administrativa de la educación pública, financiamiento público para instituciones privadas e instalación de un cuasimercado en educación, operando bajo el supuesto de que la sola fuerza del mercado lograría mejorar el sistema en eficiencia y calidad (Almonacid, 2004, 2008; Mizala y Romaguera, 2005). Durante estos primeros años, las que luego aparecerían como evidentes "fallas de mercado" -problemas de incentivos y ausencia de accountability - intentarían ser neutralizados mediante la introducción de medidas de mejora en el sistema y el incremento de insumos educativos (Mizala y

1 Coalición de partidos políticos que ganó las elecciones presidenciales entre los años 1990 y 2009. 
Romaguera, 2005; Donoso y Schmal, 2009). Es decir, guardando las proporciones: mejorado, pero más de lo mismo.

Lo que subyace a estos cambios es la pretensión de transitar del Estado maximalista de los años 70 y del minimalista de los 80, a un "Estado capaz" (Cox, 1997: 19). Un Estado que ejerciera el margen de acción permitido por el diseño institucional heredado por las fuerzas democráticas de parte de la dictadura cívico-militar, en términos de garantizar la protección del derecho a educación, aspiración inserta en las iniciativas del modernización del Estado impulsadas por entonces (Marcel, 2002). En este sentido operarían los incrementos presupuestarios y el conjunto de medidas adoptadas en términos de infraestructura, equipamientos y marco curricular, todo lo cual implicaría un enorme esfuerzo para generar el acuerdo social necesario (Corvalán, 2013), todas estas iniciativas - sin embargo como se sabe- no fueron lo suficientemente poderosas para alterar el formato básico neoliberal de la asignación de los recursos financieros y de diseño funcional del Estado en educación (Donoso, 2013).

Los resultados más relevantes del movimiento social del año 2006 pueden situarse en dos niveles: uno, en el de las ideas y otro, en el de los cambios implementados. En el primer ámbito, lo más trascendente fue que por primera vez, desde la reforma educacional de la dictadura impuesta el año 1981, se pudo discutir públicamente temas relevantes del sector, a los que la derecha y el integrismo político conservador se habían negado sistemáticamente, aprovechando el resquicio que les otorga a las minorías el sistema parlamentario binominal. De esta forma, se logra poner en entredicho la Ley Orgánica Constitucional de Enseñanza (LOCE), norma que había sido impuesta por la dictadura días previos a la instalación del nuevo gobierno democrático (marzo de 1990), la que operó como "camisa de fuerza" para controlar cualquier transformación del sistema educativo. La discusión de esta iniciativa implicó también poner en la agenda la necesidad de fortalecer la educación pública, materia que si bien posee ciertos trazos muy amplios, revaloró lo público momentos antes de su deterioro casi terminal. En razón de ello, debe incluirse la existencia y posterior informe del Consejo Asesor Presidencial para la Calidad de la Educación del años 2006 (en adelante el Consejo), generado por el gobierno como mecanismo "de solución provisional" del conflicto mencionado, instancia que inició el debate público, masivo e informado de las distintas posturas en este campo que con distintos ritmos ha transcurrido desde esa fecha.

Es en el Consejo Asesor Presidencial para la Calidad de la Educación (2006) donde se encuentran los distintos relatos en torno al problema educativo mencionado por Corvalán (2013): la centro-estatista, la de mercado, y la síntesis de ambas, encarnada por las políticas de la Concertación. Ahí se discuten los elementos de continuidad -la educación como instrumento de igualdad en sociedades desiguales, la responsabilidad del Estado en proveer acceso a la educación y por los contenidos educativos- y también los de cambio, o que representan rupturas e innovaciones, como la autolimitación de la acción estatal como proveedora, la cesión de atribuciones a actores privados y la posibilidad de lucrar. Así también se habla de transformaciones de gran escala, como la redefinición de la relación entre Estado y Sociedad respecto de la provisión educativa, mediante el paso de una institucionalidad radicada en el Estado central a los mecanismos de mercado, y el tránsito de lo fiscal a lo municipal.

En este marco fue que se generaron las condiciones sociales y políticas para sustituir, finalmente, la LOCE siendo reemplazada el año 2009 por la Ley General de Educación 
(LGE), incorporándose algunos de los aportes del movimiento del 2006 en lo operativo. Esta iniciativa, si bien puede catalogarse como un avance respecto de su antecesora, no atendió algunas materias de fondo, a saber: la relación entre la libertad de enseñanza y el derecho a una educación de calidad, no hace alusión al lucro ni impide los mecanismos indirectos de instalación del mismo (sistemas de selección de estudiantes), no atiende las demandas de cambio del sistema de financiamiento y sus instrumentos (incluyendo el copago), ni tampoco a los cambios sustantivos que se requerían en la educación superior. En esta última, salvo algunas cuestiones menores, la LOCE y la LGE son lo mismo (Donoso, 2013).

No obstante estos avances y retrocesos, el informe del Consejo es una pieza sustancial para comprender el devenir de la década y significar por tanto los cambios en la Arquitectura macro del sistema: Creación del Consejo Nacional de Educación, de la Superintendencia de Educación Escolar y de la Agencia de Calidad, instalados los dos últimos a partir del año 2012, obligando con ello a una readecuación de las funciones del Ministerio de Educación, que puede resumirse como una pérdida de su ya deteriorada capacidad de conducción de la educación nacional, fenómeno que no se compatibiliza con la aspiración de fortalecimiento de la educación pública levantada por el movimiento del 2006, siendo este el elemento clave para comprender la prolongación del conflicto hacia 2011 y la necesidad en la actualidad (2014) de atender estas materias: la nueva arquitectura de la educación pública, incluyendo en ella: el lucro en la educación, la (re) definición del rol del Estado y la selectividad/segregación al interior del sistema educativo.

\section{LOS PROBLEMAS DE LA EDUCACIÓN PÚBLICA Y SU INSTITUCIONALIDAD}

La premisa fundamenta que sustenta la necesidad de generar una nueva arquitectura de la educación pública, proviene de la constatación de que el problema central de la educación chilena se refiere a la baja calidad de la enseñanza y su estrecha asociación con la alta segregación socioeconómica y territorial del sistema educacional en su conjunto, situación que en el último cuarto de siglo se ha potenciado en razón de que el Estado no ha podido -en los hechos- asegurar el derecho a una educación de calidad a su población, al no disponer de una institucionalidad adecuada que garantice esa condición, a saber: un buen sistema nacional de educación pública.

En términos de sus detonantes fundamentales, la crisis de la educación pública no es solo ni principalmente de índole económica. Su deterioro incremental se funda, además, en su institucionalidad y en las asimétricas relaciones de competencia que debe mantener con la educación particular. Estas materias se expresan en tres componentes dominantes del sistema que deben atenderse: el modelo de financiamiento (subsidio por alumno y financiamiento compartido), la institucionalidad (municipal y regional) y el desequilibrio entre recursos asignados y las obligaciones que pesan sobre la educación pública.

Si bien existe un alto grado de consenso acerca de la necesidad de realizar transformaciones de magnitud en la arquitectura del sistema educacional, entendiendo que es una condición imprescindible para el mejoramiento sustantivo del sistema escolar público, lo cierto es que tal acuerdo no incluye consenso sobre las nuevas formas organizativas a seguir, en torno de las que hay diferencias importantes. Esencialmente, una clasificación inicial podría incluir aquellas propuestas que están por perfeccionar la institucionalidad 
actual, sin entrar en transformaciones mayores, en tanto un número importante de ideas se organizan tras una metamorfosis relevante de la arquitectura del sistema de educación pública. En razón de ello, el criterio diferenciador es seguir concibiendo la educación como un bien privado asumido como público o semipúblico (Stiglitz, 2000), en tanto las otras visiones promueven un cambio estructural con el objetivo de sacarla del mercado en consonancia con su carácter de bien público, algunas de las que la fundan explícitamente en asumirla como un derecho social.

En las visiones procambio estructural, pese a la coincidencia en que el nuevo sistema debe ser descentralizado, el debate es sobre el equilibrio entre descentralización, realidades locales y calidad de la gestión. Entonces el tema crítico es llegar a acuerdo respecto de qué tan descentralizado ha de ser el nuevo sistema, existiendo -ciertamente- visiones alternativas. Las más dominantes, efectivamente, hablan de un proceso centralizado de descentralización, es decir, dosificado desde el aparato central. En tanto las menos, definen un proceso descentralizado, con mayor autonomía de las unidades territoriales subnacionales.

Aunque pueda resultar sugestivo este debate, se trata de una discusión con escasa tradición en Chile, debido a que estas materias han sido -usualmente- relegadas a situaciones puntuales, de escaso impacto público. En nuestro medio no se ha concebido la descentralización como una política de desarrollo destinada a dar sustentabilidad económica, social y política al país, ello no forma parte de las visiones dominantes que hegemonizan los debates sobre reforma del Estado, lo que finalmente ha retardado los avances en estas materias, siendo más bien episódicos, asociados a los conflictos que van emergiendo.

\subsection{EL INFORME DE LA COMISIÓN DE MODERNIZACIÓN DE LA EDUCACIÓN (1994)}

Hacia fines del año 1994 se conoce el Informe de la Comisión de Modernización de la Educación Chilena, instancia generada por iniciativa presidencial ese mismo año, y cuyo objetivo fue identificar los principales desafíos para el sector de cara al siglo XXI. Se trató de un informe que exhibe un detallado diagnóstico y en función de ello propuestas, igualmente específica para los diversos componentes del mismo. A diferencia del Consejo del año 2006, este fue la expresión más nítida de la fuerte vinculación entre el poder político, económico y tecnocrático, ratificando lo indicado por Corvalán (2013). En este documento no se establece en ningún punto la necesidad de cambiar la institucionalidad de la educación pública, pese a que en su diagnóstico se muestran diferencia de resultados, recursos y organización que claramente evidencian el desmedro en que opera ese sector de la educación, constatando que el sector público se hacía cargo de la población más vulnerable y por ende de mayor costo agregado (Comisión de Modernización, 1994: 44-45).

Este informe da cuenta del pensamiento dominante en el área, su diagnóstico sostiene que:

En suma, nuestro análisis nos lleva a concluir que el sistema escolar chileno se ha quedado atrás con respecto a los requerimientos del país. Ofrece una cobertura amplia pero de calidad pobre y desigual; es por eso inequitativo y, además, es poco eficiente. Sus logros son, en general, insatisfactorios. Para la mayoría de los alumnos provenientes de los hogares de menores recursos, los niveles de logro son francamente malos. La formación que ofrecen los establecimientos escolares es rutinaria; no cultiva las capacidades del alumno y no enseña a 
aprender. Existen diversos programas de mejoramiento en curso que apuntan en la dirección adecuada, pero no son suficientes. La gestión del sistema, a pesar de los avances en la descentralización, es todavía inflexible. Los profesores y los alumnos trabajan en un ambiente que, en general, es poco estimulante. En esas condiciones Chile no podrá desarrollarse con fuerza en el futuro. Perderá la oportunidad histórica que tiene de convertirse en una sociedad moderna y equitativa (Comisión de Modernización, 1994: 53).

En esta visión está presente la adecuación del sistema a su arquitectura vigente, centrando las tareas sobre la escuela, sin mayores distingos si es pública o privada, en tanto reciba subvención, y poniendo énfasis en el aprendizaje con cierto énfasis en lo instrumental. El accionar de la institucionalidad debe ser en respaldar la tarea de la escuela. Esa era la idea que subyace al informe, por lo mismo no es extraño que se sostenga que "la gestión del sistema estimula y facilita la experimentación, innovación y adaptación por parte de los establecimientos, permitiéndoles definir su propio proyecto educativo y actuar con flexibilidad, responsabilidad y sujetos a adecuados mecanismos de evaluación de sus logros y desempeño" (Comisión de Modernización, 1994: 59).

Queda claro que el papel del Estado, en consonancia con lo que señala la Constitución y esencialmente la LOCE, será

otorgar especial protección al ejercicio del derecho a la educación. Con ese fin debe suministrar los recursos necesarios para asegurar la educación obligatoria de todos los niños, cuya gratuidad y calidad garantizará, en particular para quienes no están en condiciones de contribuir al financiamiento de su educación. Ningún niño chileno, por ninguna razón, puede quedar sin acceso a la educación básica. Al Estado le cabe, asimismo, fomentar el desarrollo de la educación, en particular, de la educación subvencionada, y garantizar el acceso de toda la población a un sistema educacional con formas de gestión diversificadas; otorgar, de acuerdo a la ley, el reconocimiento oficial a los establecimientos que lo soliciten y cumplan con los requisitos legales; focalizar su acción en los sectores más pobres, cuya educación debe garantizar prioritariamente, y asegurar la flexibilidad y los estímulos necesarios para que el sistema escolar en su conjunto alcance niveles crecientes de calidad, equidad y eficiencia (Comisión de Modernización, 1994: 101).

Este planteamiento sigue en el documento a la definición de Libertad de Enseñanza, como principio fundante y mandante del sistema.

En virtud de lo expuesto, es evidente que en la década de los 90 hablar de una nueva arquitectura de la educación pública no formó parte del discurso oficial ni tampoco estaba en su horizonte de debate. En el hacer de los gobiernos, al amparo de estos lineamientos y antes del movimiento estudiantil del año 2006, estaba fortalecer la escuela como centro educativo, fortalecer su autonomía, aunque sea una institución pública, y preocuparse esencialmente de los logros de aprendizaje, sin mayores distingos sobre su dependencia. El Informe de la Comisión de Modernización proveyó los fundamentos intelectuales de la LOCE: lo público implicaba en ese sentido el hacer de los privados, aunque en materia de responsabilidades ello no fuese recíproco: los empresarios privados tienen los mismos derechos financieros que los públicos, pero no los mismos deberes ante la sociedad que los públicos.

$\mathrm{Al}$ analizar estos planteamientos retrospectivamente no debiésemos haber sido sorprendidos como sociedad -como lo fuimos- por el movimiento pingüino, es más podríamos preguntarnos ¿por qué no ocurrió antes? Hay razones políticas importantes que explican 
el complejo proceso de transición a la democracia experimentado por el país (Garretón y Garretón, 2010). Si bien en el nuevo milenio se vislumbran algunos planteamientos críticos a las políticas educacionales, se trata esencialmente de académicos no insertos en círculos dominantes, como también de algunos dirigentes del gremio docente concertacionistas (Almonacid, 2004; Donoso, 2005; Picazo, 2013). Será tras el movimiento del año 2006 que se producirá el fuerte despertar de la crítica al modelo refrendada en los muchos trabajos que se han producido en los años siguientes ${ }^{2}$.

\subsection{EL INFORME DEL CONSEJO ASESOR PRESIDENCIAL PARA LA CALIDAD DE LA EDUCACIÓN} (2006)

A diferencia del informe anteriormente analizado, en el trabajo en comento se aborda con importante detalle las materias en análisis: la institucionalidad y arquitectura del sistema público de educación ${ }^{3}$, las que habían sido parte de las propuestas del movimiento estudiantil. Una segunda diferencia relevante es que esta entidad estuvo integrada por más de 100 representantes de diversos grupos y sensibilidades sobre la materia (dirigentes estudiantiles, de apoderados, de los docentes y trabajadores de la educación, especialistas, representantes de pueblos indígenas, de las iglesias, de los centros formadores de docentes, etcétera).

En la exposición del tema se consigna que los integrantes del Consejo tuvieron visiones diferentes sobre varias de las materias tratadas, plasmando con ello la diversidad de opiniones que habían salido a la luz pública gracias a este movimiento. Una parte de los integrantes considera que la institucionalidad es poco eficiente, argumentando un conjunto de razones de eficiencia social del sistema que respaldan esta visión, la que, por grupo no es vista como tal, sosteniéndose que el sistema ha sido eficiente en materia de logros de aprendizaje y de indicadores de eficiencia interna y externa del mismo, ese es el punto central de discordia a partir del que se construyen las visiones contrapuestas. El análisis de las materias de Institucionalidad, la discusión respecto de esta materia -institucionalidad y calidad, e institucionalidad y equidad- muestra las mismas tendencias discrepantes entre el grupo promodelo vigente y el más crítico de sus resultados (Cfr. Consejo Asesor Presidencial para la Calidad de la Educación ${ }^{4}$, 2006: 111-114).

No obstante, hay un consenso importante en señalar "El Estado no cuenta con herramientas para exigir mayor calidad a las instituciones educacionales o a sus proveedores, que no sea publicar los resultados del SIMCE en la prensa o verificar los requisitos antes referidos" (Consejo Asesor Presidencial para la Calidad de la Educación, 2006: 111). Lo que dará como resultado un cambio en las exigencias establecidas en la LGE para ser sostenedor educacional. Pese a estas diferencias, al analizarse específicamente la situación

\footnotetext{
2 Un ejemplo palpable de ello se puede constatar en que desde al año 2005 hasta al menos el 2007, los gobiernos de la misma coalición hablaron de la gran reforma educacional. Esta iniciativa fue "silenciada" el año 2008, saliendo del discurso oficial y desde entonces -hasta la fecha- prácticamente nadie del "establishment" se refiere a ella, la mayor pate de los textos críticos no responden a ese perfil.

3 Se expone al menos específicamente en dos capítulos (de un total de 10) representando el 15\% de la extensión del informe final.

4 Todas las referencias del informe final del Consejo Asesor Presidencial para la Calidad de la Educación citadas corresponden a la versión publicada en la web, ello porque la versión del informe final en papel posee diferencias en las páginas con la versión electrónica.
} 
de la educación hay mayor acuerdo "El Consejo concuerda que el desempeño del actual sistema de administración municipal de la educación es negativo. Hay acuerdo en un conjunto de condiciones que explican las falencias del sistema municipal, un financiamiento insuficiente para las obligaciones que poseen, disolución de responsabilidades entre el Ministerio y los municipios, escasa transferencia de competencias a las escuelas, relación desigual entre el sector municipal y el particular subvencionado" (Consejo Asesor Presidencial para la Calidad de la Educación, 2006: 115).

La revisión de algunos factores asociados a lo indicado da cuenta de tamaños muy desiguales de los sistemas comunales públicos de educación, de muchas unidades locales (municipios) muy pequeñas, con heterogeneidad de la calidad de los servicios educativos que entregan trabas legales para generar asociatividad entre los municipios, problemas de gestión de recursos, alta dependencia de los fondos financieros centrales estatales, excesiva dependencia política de las autoridades, escasa transferencia de atribuciones del Municipio a las escuelas, asimetría de responsabilidades y compromisos entre el sector particular subvencionado y el estatal (ej.: selección de estudiantes, carrera y remuneraciones docentes) y además emplazamientos territoriales diferentes, confirmando que la población más vulnerable es atendida en su derecho a educación principalmente por la educación pública (Cfr. Consejo Asesor Presidencial para la Calidad de la Educación, 2006: 114-118).

Finalmente respecto de la temática en análisis, se señala:

El Consejo estudió alternativas de organización de la educación de carácter estatal, porque está consciente que, para mejorar el desempeño general del sistema educacional, la institucionalidad del sistema de propiedad estatal es fundamental y es adecuado revisarla. Para que ésta rinda frutos se requieren, además, que los demás arreglos institucionales sean compatibles con la institucionalidad del sistema de propiedad estatal elegido (...) el Consejo ha estimado que, para estos fines, puede explorarse una modificación de la institucionalidad del sistema público. Esta debería cumplir una serie de criterios que son aplicables, tanto para orientar un nuevo diseño institucional en el cual las escuelas y liceos dejen de depender de los municipios, como para delinear un ajuste a la institucionalidad actual, que manteniendo las escuelas y liceos vinculados con los municipios, se ampare en un nuevo esquema organizacional (Consejo Asesor Presidencial para la Calidad de la Educación, 2006: 119).

Más allá del diagnóstico, el Consejo establece un conjunto de requisitos que debiese cumplir la nueva institucionalidad, cualquiera sea su forma definitiva, antes de dar cuenta de los principales aspectos referidos, se revisan los diagnósticos realizados sobre la materia por diversos estudios.

\subsection{APORTES AL DIAGNÓSTICO DE LA INSTITUCIONALIDAD DE LA EDUCACIÓN PÚBLICA}

Desde mediados de la década pasada se plantean diversos análisis sobre la institucionalidad pública que dan cuenta de la compleja situación en que se encuentra. Algunos se centran preferentemente en el diagnóstico y el mejoramiento, sin buscar otras modalidades de organización que la vigente, es decir, optimizando lo existente (Razcynski y Salinas, 2008; Marcel y Razcynski, 2009), también hay otros que presentan una crítica al exagerado optimismo en creer que "esta vuelta al Estado" permitirá resolver los problemas estratégicos de la educación pública (Picazo, 2010), otros profundizan en la 
perspectiva del Consejo (Bellei, Contreras y Valenzuela, 2010), así como también en el diagnóstico y las propuestas del informe señalado (Donoso y Arias 2011, 2013; Donoso et al., 2011; Elacqua, González y Pacheco, 2008), finalmente un debate más general se refiere al sentido de la educación pública (Atria, 2010; Bellei et al., 2010; Donoso, 2013). Tangencialmente se aludirá a los planteamientos de Beyer y Velasco (2010), Montt, Elacqua, González, Pacheco y Raczynski (2006), y Martinic y Elacqua (2010), debido a que se refieren puntualmente a estas materias.

Respecto del diagnóstico se comparte la mayor parte de los argumentos entre los distintos autores que avanzan en estas materias. Debido a la heterogeneidad de los municipios y su sistema de gestión escolar, en variables como población, tamaño y gestión de la estructura administrativa, características socioeconómicas y territoriales, se hace imprescindible corregir las asimetrías presentes, mediante una propuesta de institucionalidad pública que se ajuste por una parte a las necesidades de municipios y territorios, estableciendo los recursos requeridos, las brechas existentes y las escalas mínimas respecto a la operación normal de los municipios, y por otra, establecer una propuesta descentralizada que logre incrementar las capacidades y competencias de muchos municipios pequeños o de alta ruralidad que en general cuentan con baja generación de ingresos propios, recursos humanos (docentes) y financieros limitados y una alta dependencia del Fondo Común Municipal (Razcynski y Salinas, 2008; Marcel y Razcynski, 2009).

A estas complicaciones hay que agregar que la principal desventaja del sistema actual radica en "las fuertes limitaciones que los alcaldes tienen para cumplir su rol de sostenedores y la poca capacidad de los ciudadanos para evaluar la gestión educativa de sus alcaldes" (Elacqua et al., 2008: 153). Ello ha permitido que la principal preocupación de los alcaldes y de los encargados de la educación municipal "son los relativos a la gestión administrativo-financiera de los establecimientos, siendo los aspectos técnicopedagógicos un tema todavía secundario y minoritario, aunque emergente entre los municipios" (Razcynski y Salinas, 2009: 207).

Dentro de las condiciones adicionales para el mejoramiento de la educación se establecen, igualmente, un conjunto de condiciones que reúnen un gran consenso entre quienes han revisado estas materias, y que reflejan los problemas diagnosticados, a saber: i) revisar y alinear la normativa, estableciendo "que la educación municipal como heredera de la educación pública y garante del derecho a una educación gratuita, debe contar con similar autonomía que la particular subvencionada y someterse a similares mecanismos de acreditación. Al mismo tiempo, los sostenedores municipales tienen una responsabilidad sobre el sistema educacional y la población en edad escolar en su territorio, lo que sobrepasa la administración y gestión de los establecimientos" (Razcynski y Salinas, 2008:130); se precisa que ii) urge estudiar exhaustivamente los costos de entregar educación de calidad en distintas situaciones; iii) que se debe diseñar e implementar una política de apoyo a la expansión de las capacidades de gestión del sostenedor, que aborde su papel en la administración de los colegios en los planos administrativo, financiero y pedagógico, y su responsabilidad respecto de la planificación de la educación en su comuna, estableciendo iv) problematizar y modificar las prácticas de trabajo del Mineduc con los establecimientos educacionales y los sostenedores, y los vínculos Subdere/Mineduc, v) fomentando el trabajo en alianza con otros, precisando los propósitos que pueden tener en una red y los distintos actores que eventualmente participen, vi) incorporando de modo explícito las características del territorio en la gestión de la educación. 
En términos generales, se coincide entonces en que la crisis se manifiesta en una pérdida significativa y constante de matrícula, atribuido a las políticas de financiamiento público y para la educación privada subvencionada, a las capacidades de gestión instalada, a la institucionalidad inadecuada para la complejidad de las tareas públicas, los recursos disponibles, bajos resultados en función de las características de la población (Bellei et al., 2010: Donoso, 2013) a lo que se agrega un factor adicional planteado por Bellei et al. (2010):

La apertura de cerca de 2.000 nuevos establecimientos subvencionados particulares en los últimos años a lo largo del país -con lo que este tipo de establecimientos se ha prácticamente duplicado en solo una década-, debido a que la población en edad escolar no está creciendo, deriva en la pérdida de matrícula del sector público. Al mismo tiempo, la expansión de las escuelas particulares subvencionadas, al ir unida a refinados mecanismos académica y socialmente selectivos, ha incrementado la segregación de los estudiantes vulnerables o de bajo rendimiento, quienes tienden a concentrarse en los establecimientos públicos (Valenzuela, Bellei y De los Ríos, 2009). Este proceso aumenta la inequidad socioeconómica y de resultados académicos entre los estudiantes chilenos (p. 235).

Finalmente, Picazo (2010) pone la voz de alerta al plantear que durante las dos últimas décadas se ha estado configurando en Chile un Estado post-neoliberal, que "trata de asegurar, al mismo tiempo, cuatro objetivos principales que han atravesado la historia de la educación chilena y que han sido difíciles de conciliar: el derecho a la educación, la libertad de enseñanza, la participación de la familia y de la comunidad y el papel del Estado" (Picazo, 2010: 85), dando cuenta de la complejidad del escenario que predomina y advirtiendo "sobre la crítica del dogmatismo casi idílico de las virtudes del mercado como de las políticas que muestran una ingenua fe en el Estado para corregir distorsiones estructurales" (Picazo, 2010: 89).

\section{PROPUESTAS DE NUEVA INSTITUCIONALIDAD}

El desafío de fortalecer la educación pública implica centralmente, pero no exclusivamente, implementar un nuevo diseño institucional que dé cuenta de los principales problemas señalados: construya una propuesta de educación válida, atractiva y pertinente, que implique una formación integral de calidad, en materia de logros de aprendizaje y formación personal y ciudadana, por ende reduzca la segregación socioeconómica y académica de los estudiantes, y compense las asimetrías en la regulación del mercado educativo.

En términos de los detonantes fundamentales de la crisis de la educación pública, o se expuso, no es solo ni principalmente de naturaleza económica. Su deterioro incremental se funda, además, en su institucionalidad y en las asimétricas relaciones de competencia que debe mantener con la educación particular. Estas materias se expresan en tres componentes dominantes del sistema: el modelo de financiamiento (subsidio por alumno y financiamiento compartido), la institucionalidad (municipal, y regional) y el desequilibrio entre recursos asignados y las obligaciones que pesan sobre la educación pública.

El aspecto central será entonces definir la forma institucional más adecuada para acometer esta tarea, es decir, quién y cómo se administrarán los establecimientos públicos y las características principales que debiese tener. El Consejo estructuró un conjunto de 
recomendaciones - de al menos nueve criterios- para cualquiera de las formas institucionales por las que se opte, a saber:

- Que su única función sea la gestión de establecimientos educacionales.

- Que asuma la responsabilidad de administrar los establecimientos de propiedad del Estado (de cualquiera de sus niveles) en un territorio, que permita disponer de capacidad técnica central como también la implementación de sistemas de desarrollo profesional docente y directivo y tienda su sustentabilidad en todo plano (economías de escala).

- Que atienda en forma integrada las funciones de gestión administrativo-financiera con las de gestión técnico-pedagógica.

- Contar con un financiamiento suficiente para asegurar un adecuado funcionamiento de los establecimientos educativos según las características de educabilidad de su población.

- Compatibilizar la gestión y administración pedagógica, con la participación de la comunidad en el control de su gestión y en la definición del proyecto educativo territorial.

- Conformar un equipo ejecutivo seleccionado mediante Alta Dirección Pública.

- Supervisar y controlar a sus establecimientos, teniendo como norte fortalecer las capacidades y la autonomía pedagógica de las escuelas y liceos a su cargo.

- Respecto de las atribuciones, tener a cargo la gestión administrativa y financiera de los establecimientos, y contar con centros de costos por establecimiento; supervisar y apoyar técnica y pedagógicamente a sus establecimientos; y seleccionar y contratar al personal docente, asegurando la participación de los establecimientos en tal proceso.

- Tener la facultad de suscribir convenios con entes públicos y privados, para el cumplimiento de sus funciones (Cfr. Consejo Asesor Presidencial para la Calidad de la Educación, 2006: 119-122).

Estas orientaciones se compatibilizan en distinta forma en las alternativas que se han propuesto por los autores señalados: Consejo Asesor Presidencial para la Calidad de la Educación (2006), Elacqua et al. (2008), Raczynscki y Salinas (2008, 2009), Atria (2010), Bellei et al. (2010), Donoso et al. (2012) y Donoso (2013).

Como se ha puntualizado, si bien existe consenso acerca de la necesidad de realizar transformaciones de magnitud en la arquitectura del sistema educacional, entendiendo que se trata de una condición imprescindible para el mejoramiento sustantivo del sistema escolar público, lo cierto es que tal acuerdo no incluye consenso sobre las nuevas formas organizativas a seguir, respecto de las que hay diferencias importantes. Inicialmente están aquellas propuestas que están por perfeccionar la institucionalidad actual, sin buscar transformaciones mayores, mientras que alternativamente un número importante se organizan tras un cambio relevante de la arquitectura del sistema de educación pública. Este último grupo incluye una propuesta de volver al sistema prerreforma de 1981, es decir, todos los establecimientos públicos dependientes del Ministerio de Educación. La descripción de las modalidades se realiza brevemente debido a que ha sido latamente analizada en los trabajos citados.

\subsection{LAS PROPUESTAS "EXTREMAS O MÁS RADICALES”}

Se atribuye esta connotación debido a que suponen un escenario casi imposible de sostener o bien generar; por lo mismo, su radicalidad vendría dada por las contextos que imponen para su aplicación. 
a. "Adecuar la institucionalidad actual", defendida por algunos creyentes del sistema que, por último, reducen -esencialmente- la crisis de la educación pública a una cuestión financiera de los municipios, que se resuelve elevando el valor basal del subsidio por alumno, y a algunas materias de gestión de los recursos por parte de las autoridades locales (Sapelli, 2006; Beyer y Velasco, 2010). En síntesis, se busca el "statu quo" del actual sistema, bajo el entendido que para el caso chileno, siempre ha de existir un sector público, que aunque reducido en su tamaño, atienda aquella población más vulnerable que no representa "negocio para los privados".

b. En esta misma categoría se encuentra el caso opuesto, a saber: desmunicipalización plena, donde todos los establecimientos escolares, docentes, etcétera, vuelven a ser responsabilidad y dependencia del Ministerio de Educación. Cabe mencionar que esta situación es la que vivió el país hasta el año 1980. Esta alternativa ha sido propuesta por el gremio docente y algunos personeros de esta organización (Equipo Revista Docencia, 2011), como asimismo por dirigentes estudiantiles de educación secundaria y superior. Entendiéndose que impulsar esta iniciativa implica un cambio radical, reforma constitucional incluida, que faculte al Ministerio respectivo a asumir esta tarea, debiendo -también- reformar los otros componentes macro del sistema: la Superintendencia de Educación Escolar, la Agencia de Calidad y el mismo Consejo Superior de Educación. Eso son palabras mayores, tanto para diseñar, negociar e implementar esta reforma, como para su plena ejecución.

De igual forma implicaría volver a dotar al Ministerio de un conjunto de atribuciones legales, normativas, financieras, como también de los recursos humanos adecuados para su puesta en marcha de esta contrarreforma. Adicionalmente, sumaría en su ejecución los problemas de centralización excesiva que hoy acusan algunos ministerios que no han hecho reformas descentralizadoras. Finalmente, ello no solamente está en contra de las tendencias actuales de modernización del Estado, sino que además, es posible que implicara conflictos en algunos territorios entre el gobierno central y aquellos ciudadanos que quieren tener un papel decisional más relevante sobre aquellos derechos sociales que les competen.

\subsection{LA PROPUESTA "CONSERVADORA"}

Bajo esta categoría se identifica una variante de la propuesta inicial, a saber: "Agrupación voluntaria de municipios", conformada por aquellas comunas que han alcanzado logros educativos y operacionales importantes, permitiéndose su agrupación, se entendería que comparten un territorio, y en función de ello podría optimizar su gestión y, esencialmente generar economías de escala.

Con esta modalidad se mantendría el régimen de municipalización, también creándose estas "macrocomunas" que tendría efecto solamente para las materias educacionales, y que debería producir mayor eficiencia en la gestión de los recursos, redundado ello en el mediano plazo en el mejoramiento de la calidad de la educación. Se comprende que aquellos municipios que no cumplen los estándares de calidad requeridos para asociarse, deberían ser monitoreados -y eventualmente apoyados- directamente por la Agencia de Calidad o la Superintendencia, según sea el caso (aspectos pedagógicos la primera, normativos la segunda). 
Estas posiciones se reflejan en algunos representantes de la Asociación de Municipios, planteándose visiones diversas, algunos sugieren adecuaciones financieras que permitieran incentivar la fusión de municipios, otros mencionan la necesidad de cambiar los criterios de asignación de los recursos, estableciéndose el subsidio a partir de la matrícula efectiva de estudiantes, y no del complejo sistema vigente de asistencia promedio diaria/mes por estudiante. El gran motor de esta propuesta es la posibilidad de generar economías de escala a partir de alcanzar tamaños de unidades territoriales mayores y desde allí impactar los aprendizajes de los estudiantes. De esta manera, más bien el tema es visto desde las ventajas que representa para la gestión institucional una iniciativa como esta, más que por su impacto sobre la calidad de la educación. En razón de ello, los argumentos de optimización de los recursos y de generación de economías de escala, son fundamentos más bien insuficientes para sostener una transformación de esta naturaleza.

\subsection{LAS PROPUESTAS "DE AVANZADA"}

En esta categoría se encuentran dos propuestas, la primera de ellas con ciertos matices en su formulación:

a. Servicio Nacional de Educación descentralizado", dependiente del Ministerio de Educación. La idea en torno de la creación de un Servicio Nacional descentralizado, es que el Estado vuelva a hacerse cargo en forma directa o indirecta de los establecimientos mediante corporaciones que agrupan a municipios, es una propuesta, dependiente del gobierno central o de los gobiernos subnacionales. Ciertamente, cada alternativa tiene pros y contras que exigen una implementación cuidadosa y responsable de políticas complementarias (CEPPE, 2011).

b. Las Corporaciones Territoriales de Educación, sin dependencia del Ministerio, sino de los gobiernos regionales respectivos, es decir, plenamente descentralizados. Esta discusión refleja las diversas opiniones acerca de cuanta descentralización debería soportar el sistema. Esa es la diferencia sustantiva que subyace a este debate. En la primera alternativa se plantea descentralización, pero bajo el nexo funcional del Ministerio de Educación central. Mientras que en la segunda propuesta no se sostiene este nexo, se deja a los gobiernos subnacionales con la responsabilidad de impulsar esta iniciativa, y su relación con el Ministerio y las restantes instituciones será igual a la de un sostenedor privado, no existiría razón alguna para generar un trato diferente en este plano.

Ambas visiones comparten un conjunto de elementos que les diferencian de las propuestas anteriores. Los servicios gozarían de un grado importante de autonomía legal, financiera y operacional. Su estructuración como Corporaciones de Derecho Público, dedicadas exclusivamente a estos fines, no solamente garantiza esta tarea, sino que otorga mayor agilidad en los ámbitos de gestión señalados. En razón de ello se trataría de un servicio público, que bajo la figura de un gobierno superior colegiado, regido por autoridades técnicas, en lo pedagógico y de gestión misma, y no de confianza política.

La nueva institucionalidad necesita constituir un organismo técnico especializado, con giro único en educación, coherente con políticas de desarrollo, debiendo contar con capacidades administrativas diseñadas en función de lo educativo, dotada para gestionar de manera adecuada recursos emanados del nivel central (Raczynski, 2012). 
También hay semejanzas en la necesidad de cambiar el sistema de financiamiento por un proceso que dé estabilidad de recursos y retrate correctamente el complejo fenómeno que se busca financiar, algo que el sistema vigente no logra. Al respecto hay algunas diferencias que se explican más adelante. También se concibe que estas unidades de gestión de la educación pública debiesen tener un acento territorial, lo que ha de combinarse con criterios flexibles de agrupación, es decir, con tamaños de corporaciones diferentes.

Un segundo tipo de coincidencias entre ambas visiones es que la desmunicipalización no ha de ser el único objetivo de las reformas al sistema educacional. Resulta tan relevante como lo señalado corregir las asimetrías de la actual normativa entre escuelas públicas y particular-subvencionadas, que dice relación con deberes y derechos muy asimétricos entre ambos tipos de sostenedores. Ello debiese considerar en el mediano plazo el fin del copago (financiamiento compartido) y de la selección de alumnos en la educación privada, y por cierto establecer sus implicancias en el ya señalado cambio del sistema de financiamiento para la educación pública.

\section{PUNTOS CRÍTICOS QUE SUBYACEN A LA DEFINICIÓN DE LA NUEVA INSTITUCIONALIDAD}

Para avanzar en este campo, construyendo una política de Estado al respecto, es necesario un mayor debate que permita dilucidar conflictos y construir consensos en torno de algunas materias que requieren de definiciones claras, debido a que se trata de aspectos claves o puntos críticos vitales para el desarrollo de la nueva institucionalidad, y que en tanto no se llegue a definiciones que satisfagan los diagnósticos reseñados se corre el riesgo de que estos no logren conformar una solución efectiva del problema.

\subsection{DEFINIR QUÉ SE ENTENDERÁ POR EDUCACIÓN PÚBLICA}

Aunque resulte extraño, en el caso chileno se ha elaborado una intencionada confusión acerca de qué es educación pública, y su definición está siendo amenazada por la ambigüedad, en razón de que algunos la establecen como toda aquella que recibe subvención del Estado, independiente de la propiedad del ejecutor o sostenedor. Con esta argumentación los seguidores del neoliberalismo relativizan "lo público" e igualan -por cierto para algunos efectos- el hacer de los privados con lo público, de más está decir que en materia de responsabilidades no son equivalentes, pero eso no es considerado por quienes sustentan tal intencionada simetría.

La diferencia entre lo público y lo privado en la educación escolar vendría dada porque los primeros no ponen ninguna restricción al ingreso de estudiantes en tanto los segundos sí lo hacen (Atria, 2010). No obstante esta definición puede ser restrictiva (Donoso, 2013) y por ende resulta indispensable incorporar otros factores que definen la educación pública: (i) el desarrollo (de tipos) de proyectos educativos considerados de interés común, (ii) la garantía de la existencia de proyectos educativos no particularistas o excluyentes, (iii) la garantía del derecho constitucional de todos (a nivel social y geográfico) a una educación obligatoria y gratuita, y iv) la fijación de un estándar práctico de calidad para el conjunto del sistema escolar, promoviendo la búsqueda de 
la excelencia en sus escuelas y, por extensión, en las escuelas privadas (Bellei et al., 2008, 2010).

Desde el punto de vista económico, esta discusión implica consensuar la definición de un bien público, como aquel que produce beneficios indiscriminados (sin mayores restricciones que los recursos disponibles), que se comparten entre un amplio conjunto de personas, hayan o no pagado los costos de su producción (Donoso y Schmal, 2009). Lo que finalmente implica que cada sociedad debe definir "aquellas materias de interés público", lo que se traduce en un acuerdo o pacto social que considera aquello que ha de ser administrado por incentivos de mercado y cuáles no deben seguir esa orientación. Materias que el neoliberalismo cree haber resuelto declarándose como independiente del razonamiento moral, cuestión que en los hechos, por cierto, es imposible.

Toda esta discusión considera dos supuestos claves que deben -igualmente dilucidarse-: el primero respecto de un cambio del rol del Estado, de uno subsidiario a un Estado de derechos sociales. En segundo lugar, asociado a lo anterior, tomar la decisión -en forma democrática- si van a existir áreas que no sean reguladas por el mercado y en razón de ello cuáles serían (Sendel, 2012).

Esta transformación del rol del Estado se entiende como un proceso paulatino, pero consistente, ha de implicar reconquistar su lugar como principal proveedor y actor de referencia de la educación, transformándose en un Estado garante de derechos sociales. Ello significa centrar su papel en la provisión de educación pública de alta calidad, recuperando su tarea de guía y referente de calidad para todo el sistema. El eje estructurador de esta segunda visión es traspasar la tuición administrativa de la educación escolar de los alcaldes (municipios) a una institucionalidad pública descentralizada, hoy en debate sus distintas figuras (Bellei et al., 2010).

Estas materias no han sido resueltas a la fecha por el magro debate sobre la nueva institucionalidad, entonces, más allá del diagnóstico del problema, si no hay acuerdo en estos puntos el avance será claramente menor.

\subsection{LA LIMITACIÓN DE ARTICULAR LOS CAMBIOS CON LA INSTITUCIONALIDAD EXISTENTE}

Hay un punto neto de diferencia entre las propuestas que no ha sido debidamente dilucidado. En el informe del Consejo Asesor Presidencial para la Calidad de la Educación (2006), en el estudio de Elacqua et al. (2008), en el trabajo de Beyer y Velasco (2010) y en los trabajos de Raczynski y Salinas (2008, 2009) y Raczynski (2012), se insiste mucho en la necesaria compatibilidad de los cambios con el escenario institucional vigente, ello por cierto introduce un factor de mayor factibilidad a las propuestas, pero también, y para estos efectos es muy importante, limita las opciones de cambio a esta condicional, evitando de esta forma llegar a propuestas de transformaciones más profundas, siendo un punto importante a debatir que debiera clarificarse, esto es, si se va a hacer una modificación compatible con lo vigente, o bien, si va a existir una opción de mayor cambio, por incremental que fuese este proceso, los pasos a seguir han de estar bien delimitados.

Insistir en la propuesta de alta compatibilidad con la institucionalidad vigente es centrarse esencialmente en la articulación de lo local como base de toda solución, y en cierta forma no comprender la necesidad imperiosa de desalcaldizar la educación pública 
y desmunicipalizar su gestión. Si esta cuestión no es adecuadamente controlada, es posible que las soluciones provisionales, que en muchas oportunidades suelen ser definitivas, terminen agravando el problema en vez de solucionarlo, pues finalmente ello derivará en trabajar con macrocomunas, pero bajo una institucionalidad muy similar a la actual, lo que puede resultar un contrasentido.

\section{3. ¿CUÁNTO DESCENTRALIZAR?}

En las visiones procambio estructural, pese a la coincidencia en que el nuevo sistema debe ser descentralizado, el debate es sobre el equilibrio entre descentralización, realidades locales y calidad de la gestión. Entonces el tema crítico es llegar a acuerdo respecto de qué tan descentralizado ha de ser el nuevo sistema, existiendo -ciertamente- ideas alternativas.

En la propuesta del CEPPE (2011) se aborda esta problemática en forma directa, en el Informe del Consejo se analiza aunque no se propone una opción definitiva. El tema crítico es llegar a acuerdo respecto de qué tan descentralizado ha de ser el nuevo sistema, existiendo -ciertamente- visiones alternativas.

Las ideas dominantes hablan de un proceso centralizado de descentralización, es decir, dosificado desde el aparato central. En tanto las menos definen un proceso descentralizado, con mayor autonomía de las unidades territoriales subnacionales. Por ello es que se propone un servicio nacional, como un ente que coordine a los corporaciones públicas descentralizadas. Pareciera que infunde cierto temor en algunos grupos de técnicos el que existan entidades regionales o subregionales, con autonomía, y que no tengan una vinculación con el aparato central. Ello resulta extraño porque en el diseño actual no está concebido de esa manera, si bien puede ser una falencia, en la práctica no lo es, debido a que no existe un vínculo con el aparato central, sino porque los entes subcentrales de un mismo territorio no están debidamente articulados. De existir un servicio nacional para la Educación Pública, debiese haber uno equivalente para la particular, lo que no sería fácil de comprender ni menos de implementar, y de aceptarse esta iniciativa, ¿qué sentido tendrían las Secretarías Regionales de Educación, y qué rol desempeñarían las unidades provinciales?

Aunque pueda resultar sugestiva esta línea argumentativa, se trata de un debate con exigua tradición en Chile, pues estas materias han sido usualmente relegadas a aspectos puntuales, de escaso impacto público. No se ha concebido en el país la descentralización como una política de desarrollo destinada a dar sustentabilidad económica, social y política al mismo país, ni tampoco forma parte de las visiones dominantes que hegemonizan los debates sobre reforma y modernización del Estado. Por el contrario, son más bien materias de orden menor, admitidas más como un resabio de política social, usualmente se habla de desconcentración y en oportunidades se le hace erróneamente sinónimo de descentralización.

Para avanzar en esta discusión país, necesitamos clarificar el camino de cuánta descentralización se incorporará al sistema y de las razones que sustentan esta decisión. Una buena parte del nivel ineficiencia que ha venido mostrando el Estado nacional, corresponde a una sobredemanda de respuestas ante una institucionalidad que está sobrepasada en sus capacidades. Cuestión que se ha agravado porque los conflictos cada vez tienen mayor carga de expresión local, ante la que el excesivo centralismo del Estado chileno, 
lento para responder a estas crecientes demandas de escala subnacional, tampoco -por lo general- logra entenderlas, debido a la desestimación de esta dimensión en el mismo diseño político del Estado.

En la discusión pública chilena, lo subnacional es un espacio muy reducido, aún con problemas de validación que permitan construir políticas más autónomas desde esta perspectiva. En el país, algunas regiones que han caminado en este sentido, sin embargo, no han sido usualmente los ciudadanos de regiones quienes han reclamado por gobiernos subnacionales poderosos, tendencia que se comienza a quebrar en forma paulatina desde hace un tiempo. En la actualidad son estos ciudadanos quienes denuncian las incoherencias entre los diversos niveles del Estado, entre las políticas sectoriales y las territoriales. Es decir, se reclama un Estado que dé gobernabilidad a las políticas subnacionales, tras grandes debilidades normativas, políticas y de identidad del Estado subsidiario vigente.

\subsection{CAMBIAR LOS PRINCIPIOS E INSTRUMENTOS DE FINANCIAMIENTO}

Aunque se ha reconocido que la crisis de la educación pública no es solo ni principalmente de naturaleza económica, uno de los aspectos insoslayables de abordar es el cambio del modelo de financiamiento que con arreglos secundarios, más o menos eficientes, viene operando masivamente desde 1981. A lo que debería sumarse las transformaciones en la arquitectura del sistema, y también la implementación de políticas efectivas de reducción del desequilibrio entre recursos asignados y las obligaciones que pesan sobre la educación pública, que incide en las oportunidades educativas que se proveen.

Para encauzar la solución a la crisis de la educación pública es imprescindible cambiar el modelo, sus principios y los instrumentos de financiamiento, por un sistema que refleje la complejidad del fenómeno educativo en su conjunto que se pretende financiar. Con "la complejidad del fenómeno" se entienden las metas de calidad, las condiciones con las que se trabaja, los recursos disponibles para alcanzar esas metas.

La discusión de estas materias siempre provoca mucha tensión en la sociedad chilena, más aún cuando la temática del lucro en educación es una materia tan vigente y alerta, que pareciera estar muy lejos de poder alcanzarse un consenso razonable al respecto (Donoso y Alarcón, 2012). Debemos comprender que en este sistema hay sostenedores que persiguen distintos fines, por lo mismo, lo primero es sincerar las finalidades perseguidas y conforme su aceptación, dar debido cauce de responsabilidades y compromisos a quienes buscan lucrar respecto de aquellos que dicen no lucrar. Un sistema tan desregulado como el chileno, que ha operado con un libertinaje indescifrable por más de tres décadas no es difícil normarlo, pero sí es imprescindible. Para evitar avanzar en este sentido, algunos señalan que toda regulación tiende a "nivelar hacia abajo", lo que está lejos del ánimo de lo que en este caso se expone. El sentido de nivelar es únicamente ofrecer oportunidades de calidad real para todos, y no que existan discriminaciones abiertas u ocultas que no respondan a este principio.

Ciertamente la provisión de oportunidades tiene estrecha aunque no exclusiva vinculación con los recursos financieros “...el tema principal de los debates sobre educación a nivel global es su costo. De ahí la renuencia a afirmar que la educación es un derecho humano y el silencio acerca de los costos de no brindarla" (Tomasevski, 2004: 13). No obstante el punto de inflexión clave para enfrentar esta temática es comprender que el financiamiento público y la educación pública deben ser consistentes con el objetivo 
inicial de: romper el origen de cuna y territorial que determinan gran parte de los resultados educativos del sistema chileno. Es decir, que la educación provea efectivamente de un valor agregado a sus estudiantes que permita reducir el impacto negativo de las diferencias de origen de capital social, económico y territorial.

Esto implica debatir nuevos principios sustentados en la decisión de que la educación no sea regida por los principios de mercado. Tras esta decisión hay un conjunto de instrumentos que han probado ser eficientes para la tarea que se busca acometer, cuya implementación, más allá de la transición entre el modelo actual y el nuevo, no debería ser tan compleja.

\subsection{REDUCIR EL DESEQUILIBRIO ENTRE LAS OBLIGACIONES DE LA EDUCACIÓN PÚBLICA CON LA PRIVADA SUBSIDIADA}

Todas las medidas de mejoramientos de la educación pública que se han señalado, en diverso grado, son relevantes, pero insuficientes si, en forma concomitante no se equilibran deberes y derechos del sector público con el particular subvencionado. Como se mencionó, este proceso no es "nivelar hacia abajo", sino por el contrario garantizar la equidad del acceso a las oportunidades educativas.

De no implementarse medidas en este plano, la educación pública aumentará su sobrevida, pero finalizará, un poco más tarde como un sector que atiende los grupos marginales que nadie del sector privado quiere o puede atender. Como se ha sostenido en diversos textos, el derecho a educación en Chile lo garantiza solamente la educación Pública (Reddondo, 2007; Atria, 2010; Donoso, 2013). Esta afirmación se sustenta en que los sostenedores privados, argumentando diferencias entre el proyecto educativo del establecimiento y la adhesión al mismo que tendrían los padres, se puede producir la no selección de un potencial estudiante, cuestión que en los hechos ocurre con frecuencia, amparados en el derecho de propiedad del sostenedor, y no el derecho a educación que sustenta la Constitución. De igual forma, existe un conjunto de atribuciones de los privados en materia de régimen de contrato y de garantías salariales que hacen incomparable las obligaciones de los sostenedores públicos respecto de los privados. En los primeros, las obligaciones son muy superiores a los privados, lo que se trataría sería que cualquiera fuese el empleador, en tanto las remuneraciones debiesen tener un régimen similar. También hay obligaciones de rendición de cuentas de la gestión técnico-pedagógica y también financiera que marcan diferencias entre ambos sectores que deberían igualarse.

Otros asuntos como el régimen de copago, requieren de una atención mucho mayor para no generar la desprotección de los estudiantes y garantizar que los resultados educacionales puedan alcanzarse.

Todo el análisis de estos puntos requiere clarificaciones previas sobre las obligaciones de quienes reciben subsidio del Estado, los compromisos que implican y las atribuciones de los privados para operar con estos recursos y en razón de ello extender el derecho de propiedad hacia los ámbitos educacionales. Cuesta entender que recursos financieros que tienen el mismo origen, sentido, monto y finalidad puedan implicar compromisos diferentes según el propietario del establecimiento, algo insólito pues los dineros se proveen para una finalidad educativa y no del empresario. 


\section{REFERENCIAS BIBLIOGRÁFICAS}

Almonacid, C. (2008). La educación particular subvencionada como cooperadora del Estado. En C. Bellei, D. Contreras y J. P. Valenzuela (Eds.), La agenda pendiente en educación. Profesores, administradores y recursos: Propuestas para la nueva arquitectura de la educación chilena (pp. 156-179). Santiago: Universidad de Chile-UNICEF.

(2004). Un cuasi mercado educacional: La escuela privada subvencionada en Chile. Revista de Educación, (333), 165-196.

Atria, F. (2010). ¿Qué educación es "pública"? En C. Bellei, D. Contreras y J. P. Valenzuela (Eds.), Ecos de la Revolución Pingüina. Alcances, debates y silencios en la reforma educacional (pp. 153-182). Santiago: Universidad de Chile-UNICEF.

Bellei, C., Contreras, D. y Valenzuela, J. P. (2010). Viejos dilemas y nuevas propuestas en la política educacional chilena. En C. Bellei, D. Contreras y J. P. Valenzuela (Eds.), Ecos de la Revolución Pingüina. Avances, debates y silencios de la reforma educacional (pp. 11-28). Santiago: Universidad de Chile-UNICEF.

. (2008). Debates sobre la educación chilena y propuestas de cambio. En C. Bellei, D.

Contreras y J. P. Valenzuela (Eds.), La agenda pendiente en educación. Profesores, administradores y recursos: Propuestas para la nueva arquitectura de la educación chilena (pp. 13-45). Santiago: Universidad de Chile-UNICEF.

Beyer, H. y Velasco, C. (2010). Una educación pública más efectiva. ¿Los árboles no dejan ver el bosque? En C. Bellei, D. Contreras y J. P. Valenzuela (Eds.), Ecos de la Revolución Pingüina. Avances, debates y silencios de la reforma educacional (pp. 183-224). Santiago: Universidad de Chile-UNICEF.

CEPPE (2011). ¿Qué hacer con la educación municipal? Santiago: Centro de Estudios de Políticas y Prácticas en Educación - Centro de Políticas Públicas UC.

Comisión Nacional para la Modernización de la Educación (1994). Los desafios de la educación chilena frente al siglo XXI. Santiago: Editorial Universitaria.

Consejo Asesor Presidencial para la Calidad de la Educación (2006). Informe final del Consejo Asesor Presidencial para la Calidad de la Educación. Recuperado el 10 de enero de 2014 desde http://mt.educarchile.cl/MT/jjbrunner/archives/libros/ConsejoAsesor/Inf_def.pdf

Corvalán, J. (2013). La narrativa educacional chilena y su proceso de transformación reciente: Un análisis sociológico-histórico. Folios. Segunda Época, (37), 63-81.

Cox, C. (1997). La reforma de la educación chilena: Contexto, contenidos, implementación. Santiago: PREAL.

Donoso, S. (2013). El derecho a educación en Chile. Nueva ciudadanía tras el ocaso neoliberal. Santiago: Bravo y Allende Editores.

y Arias, Ó. (2013). Desplazamiento cotidiano de estudiantes entre comunas de Chile: Evidencia y recomendaciones de política para la nueva institucionalidad de la educación pública. EURE. Revista Latinoamericana de Estudios Urbanos Regionales, vol. 39 (116), 39-75.

y Alarcón, J. (2012). El lucro en la educación chilena: Debate conceptual acerca del sentido de la educación pública y de la privada. Pro-Posições, vol. 23 (2), 33-50.

, Castro, M. y Davis, G. (2012). Visiones dominantes de la política educacional en la transición chilena hacia la democracia (1991-2010). Jornal de Políticas Educacionais, (12), 57-69. y Arias, Ó. (2011). Diferencias de escala en los sistemas de educación pública en Chile.

Ensaio: Avaliação e Políticas Públicas em Educação, vol. 19 (71), 283-306.

, Cancino, V., Arias, Ó., Castro, M., Davis, G., Benavides, N. y López, L. (2011). La educación pública abandonada por el Estado: Los municipios ante la encrucijada del futuro. En J. Gairin y S. Sánchez (Eds.), Municipio y educación: Reflexiones, experiencias y desafíos (pp. 35-50). Santiago: FIDECAP.

- y Schmal, R. (2009). Introducción a la economía de la educación. El fenómeno educativo y su connotación económica. Talca: Editorial Universidad de Talca. 
(2005). Reforma y política educacional en Chile 1990 - 2004: El neoliberalismo en crisis. Estudios Pedagógicos, vol. 31 (1), 113-135.

Elacqua E., González, S. y Pacheco, P. (2008). Alternativas institucionales para fortalecer la educación pública. En C. Bellei, D. Contreras y J. P. Valenzuela (Eds.), La agenda pendiente en educación. Profesores, administradores y recursos: Propuestas para la nueva arquitectura de la educación chilena (pp. 134-155). Santiago: Universidad de Chile-UNICEF.

Equipo Revista Docencia (2011). Aportes para el debate: Institucionalidad de la educación pública. Revista Docencia, (44), 34-47.

Garretón, M. A. y Garretón, R. (2010). La transición incompleta en Chile: La realidad tras los rankings internacionales. Revista de Ciencia Política, vol. 30 (1), 115-148.

Marcel, M. y Razcynski, D. (2009). La asignatura pendiente. Claves para la revalidación de la educación pública de gestión local en Chile. Santiago: Uqbar Editores.

(2002). Las opciones de reforma del Estado. En S. Valdés (Ed.), Reforma de Estado.

Volumen II: Dirección pública y compras públicas (pp. 213-28). Santiago: Centro de Estudios Públicos.

Martinic, S. y Elacqua, G. (Eds.). (2010). ¿Fin de un ciclo? Cambios en la gobernanza del sistema educativo. Santiago: UNESCO-PUC.

Mizala, A. y Romaguera, P. (2005). Calidad de la educación chilena: El desafío de la próxima década. En P. Meller y O. Landerretche (Eds.), La paradoja aparente. Equidad y eficiencia: Resolviendo el dilema (pp. 63-407). Santiago: Taurus.

Montt, P., Elacqua, G., González, P., Pacheco, P. y Raczynski, D. (2006). Hacia un sistema escolar descentralizado, sólido y fuerte. El diseño y las capacidades hacen la diferencia. Santiago: MINEDUC.

Picazo, I. (2013). Las políticas escolares de la Concertación durante la transición democrática. Santiago: Ediciones Universidad Diego Portales.

(2010). La metamorfosis de la regulación pública en la educación escolar en Chile: Hacia un estado post-neoliberal. Revista Pensamiento Educativo, vol. 46 (1), 63-91.

Redondo, J. (Coord.). (2007). El derecho a la educación en Chile. Buenos Aires: Fundación Laboratorio de Políticas Públicas en Educación.

Raczynski, D. (2012). Realidad de la educación municipal en Chile: ¿Liderazgo del sostenedor municipal? En J. Weinstein y G. Muñoz (Eds.), ¿Qué sabemos sobre los directores de escuela en Chile? (pp. 181-217). Santiago: Fundación Chile - Pontificia Universidad Católica de Chile.

y Salinas, D. (2009). ¿Cómo se hace realmente gestión educativa municipal? En M. Marcel y D. Razcynski (Eds.), La asignatura pendiente. Claves para la revalidación de la educación pública de gestión local en Chile (pp. 177-308). Santiago: Uqbar Editores.

. (2008). Fortalecer la educación municipal. Evidencia empírica, reflexiones y líneas de propuesta. En C. Bellei, D. Contreras y J. P. Valenzuela (Eds.), La agenda pendiente en educación. Profesores, administradores y recursos: Propuestas para la nueva arquitectura de la educación chilena (pp. 105-133). Santiago: Universidad de Chile - UNICEF.

Sapelli, C. (2006). Desafíos del sistema educativo: La subvención preferencial. Temas de Agenda Pública, vol. 1 (1), 1-8.

Sendel, M. (2012). Lo que el dinero no puede comprar: Los límites morales del mercado. Barcelona: Random House Mondanari.

Stiglitz, J. (2000). La economía del sector público. Madrid: Antonio Bosch Editor.

Tomasevski, K. (2004). El asalto a la educación. Barcelona: Intermón Oxfam Editorial. 